\title{
Respiratory Motion Influence on Catheter Contact Force During Radio Frequency Ablation Procedures
}

\author{
Martin Koch $^{a}$, Alexander Brost ${ }^{b}$, Joachim Hornegger ${ }^{a, c}$, Norbert Strobel ${ }^{d}$ \\ ${ }^{a}$ Pattern Recognition Lab, Friedrich-Alexander-Universität \\ Erlangen-Nürnberg (FAU), Erlangen, Germany \\ ${ }^{b}$ Department of Radiology, Stanford University, Stanford, CA, USA \\ ${ }^{c}$ School in Advanced Optical Technologies (SAOT), Erlangen, Germany \\ ${ }^{d}$ Siemens AG, Healthcare Sector, Forchheim, Germany
}

\begin{abstract}
Minimally invasive catheter ablation is a common treatment option for atrial fibrillation. A common treatment strategy is pulmonary vein isolation. In this case, individual ablation points need to be placed around the ostia of the pulmonary veins attached to the left atrium to generate transmural lesions and thereby block electric signals. To achieve a durable transmural lesion, the tip of the catheter has to be stable with a sufficient tissue contact during radio-frequency ablation. Besides the steerable interface operated by the physician, the movement of the catheter is also influenced by the heart and breathing motion - particularly during ablation. In this paper we investigate the influence of breathing motion on different areas of the endocardium during radio frequency ablation. To this end, we analyze the frequency spectrum of the continuous catheter contact force to identify areas with increased breathing motion using a classification method. This approach has been applied to clinical patient data acquired during three pulmonary vein isolation procedures. Initial findings show that motion due to respiration is more pronounced at the roof and around the right pulmonary veins.
\end{abstract}

Keywords: Radiofrequency Ablation, Contact Force, Respiratory Motion, Electrophysiology

\section{MOTIVATION}

Radio frequency (RF) ablation is a common treatment option for atrial fibrillation (Afib) [1]. Afib is a heart arrhythmia affecting the left atrium. In contrast to the regular sinus rhythm, irregular electric signals circle within the left atrium. For paroxysmal Afib, the isolation of the pulmonary veins, attached to the left atrium, is the main goal [2]. Ablation lesions need to be placed on the endocardium of the left atrium to block the electric signals. These lesions need to be transmural and form a contiguous line to ensure durable signal block [3]. The actual effective ablation energy per lesion may, however, not only depend on the RF generator settings but also on their respective position. There are several motion patterns present during the intervention that affect an ablation site. Besides overall patient motion, cardiac and respiratory motion also effect the position and deformation of the left atrium [4]. During regular breathing a mean movement of the pulmonary veins of about $1.9 \mathrm{~cm}$ has been reported [5]. This movement also affects the RF ablation catheter. Klemm et al. investigated the catheter motion due to heart beat and respiration at different positions around the left atrium, with respiration as the major source of relative motion [6]. This study was performed using a 3-D mapping system.

Catheter ablations are commonly performed, at least in part, under fluoroscopic guidance. Fluoroscopic images for a cardiac ablation procedure are shown in Figure 1 for a bi-plane X-ray system. Regular X-ray imaging offers low soft-tissue contrast, therefore new approaches using

Send correspondence to Martin.Koch@cs.fau.de 


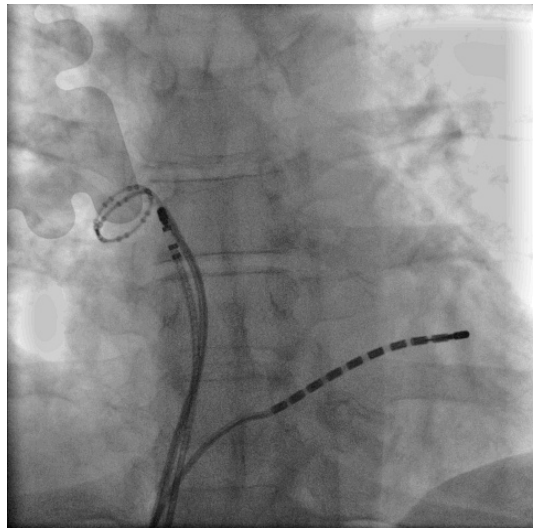

(a)

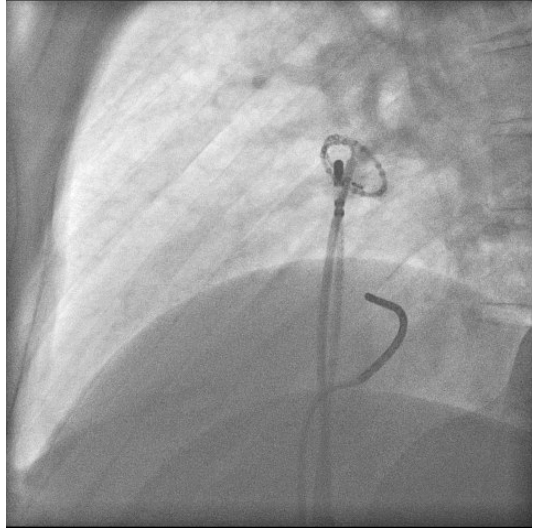

(b)

Figure 1. Fluoroscopic images of a bi-plane X-ray system during cardiac ablation procedure. The left image (a) shows the anterior view, and the right image (b) the lateral view.

augmented reality features have been proposed [7]. These systems overlay patient specific heart models extracted from pre-procedural 3-D data sets onto live fluoroscopy [8]. In the context of augmented fluoroscopy, motion compensation of the overlay image has become increasingly important as well. Brost et. al presented a method based on circumferential mapping catheter tracking $[9,10]$. Other approaches use the coronary sinus catheter as reference for motion compensation [11].

Recent developments introduced RF ablation catheters capable of measuring the contact force at the catheter tip [12]. Clinical benefits of contact force measurements have been stated in first studies, e.g. increased procedural efficacy during pulmonary vein isolation [13]. We previously described an integrated augmented fluoroscopy system with contact force assessment, that combines the 3-D localization capabilities of a navigation system with the novel contact force information $[14,15]$. The benefit of the combined system is that the measured force signal can be mapped to a 3-D location. The contact force is measured continuously, during mapping as well as during ablation. The contact force signal provides information about motion present within the left atrium, which might not be observable otherwise. In particular, our goal in this paper is to find out how respiratory motion is reflected in the contact force data and how it is affected by different spatial position.

\section{METHODS}

During the RF ablation procedure, the catheter contact force was recorded for each ablation point together with the 3-D location of the catheter tip. The 3-D location of the catheter tip was reconstructed semi-automatically, by selecting the tip in two orthogonal 2-D projections. For each ablation lesion one 3-D catheter position was recorded. The assumption of fixed catheter position for each lesion is based on a point-wise ablation strategy. The 1-D contact force signal measured at the catheter tip is sampled with a sampling rate $f_{s}$. Tracked ablation points of a pulmonary vein ablation procedures with the corresponding patient specific mesh model is shown in Figure 2.

To determine the influence of the respiratory motion on the catheter tip, we analyzed the catheter tip-to-tissue contact force during RF ablation. The signal is transformed from time domain to frequency domain for further computations. Processing of electrocardiograms (ECGs) is mostly done in frequency or time-frequency domain, e.g. extraction of respiratory frequency from ECGs in the analysis of heart rate variability [16]. 


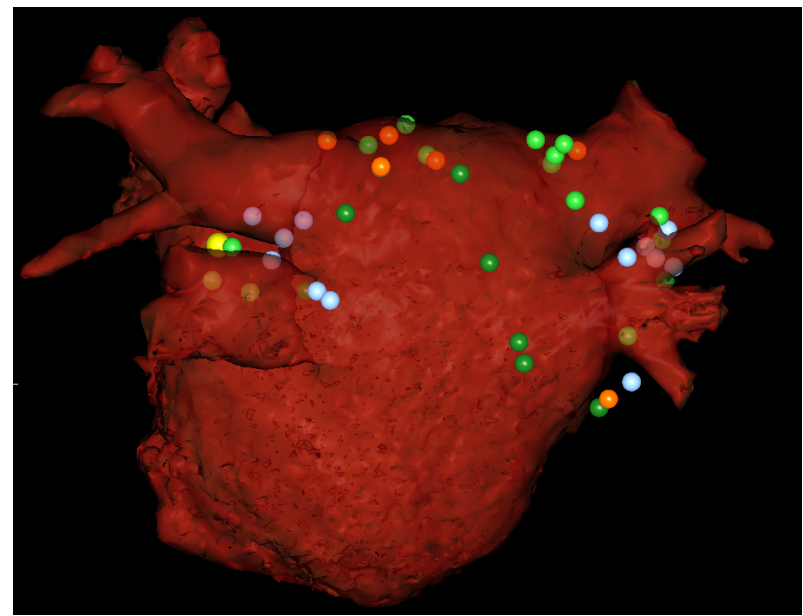

Figure 2. Patient specific mesh model of left atrium with tracked RF ablation points. The mesh model was extracted from a pre-procedural 3-D CT data set. The ablation points were reconstructed from 2-D projections.

The regular breathing frequency is between 10 and 20 breaths/min $(0.17$ and $0.33 \mathrm{~Hz}$ respectively) [17]. Therefore, a simple bandpass filter was applied to the contact force signal in the frequency domain to extract the respiratory frequencies.

For each ablation point $m=1 \ldots M$, a vector of discrete contact force samples $\mathbf{y}^{m}=$ $\left[y_{1}^{m}, \ldots, y_{L^{m}}^{m}\right]$ was acquired at a sampling rate $f_{s}$ of $10 \mathrm{~Hz} . M$ determines the total number of ablation points. The length of each vector $L^{m}$ varies, and depends on the corresponding ablation duration. The frequency domain representation $\hat{\mathbf{Y}}^{m}$ is computed via discrete fast Fourier transform. The frequency components are divided by the length of the corresponding sample vector $\mathbf{Y}^{m}=\hat{\mathbf{Y}}^{m} / L^{m}$. A sample contact force signal with corresponding Fourier transform is shown in Figure 3.

We define $p^{m}$ as the cumulated magnitude of frequencies from $f_{\text {low }}=0.17 \mathrm{~Hz}$ to $f_{\text {high }}=$ $0.33 \mathrm{~Hz}$,

$$
p^{m}=\sum_{i=i_{l}}^{i_{h}}\left\|Y_{i}^{m}\right\|,
$$

where $i_{l}$ and $i_{h}$ represent the discrete indices corresponding to $f_{\text {low }}$ and $f_{\text {high }}$ respectively. The cumulated magnitude $p^{m}$ can be interpreted as an index for the impact of respiratory motion on the corresponding ablation point. Higher values depict strong breathing motion, whereas low values indicate minor breathing motion. By not considering the DC component in our feature, we effectively subtract the mean, i.e., the average force. This makes our feature independent of the actual force applied. To determine points with high respiratory motion influence, a simple threshold classifier was applied. The threshold $\lambda_{t h}=5.0$ was determined empirically.

\section{EVALUATION AND RESULTS}

The feature $p^{m}$ was computed for each ablation point separately. Points with high respiratory motion influence were added to Group (I), the remaining points were assigned to Group (II). Based on this clustering, additional parameters related to contact force were calculated for each ablation point and evaluated per group. The additional parameters evaluated were average contact force per ablation point, force-time-integral (FTI) [18] and ablation duration per point. Based on the 3-D position of the ablation point, evaluation in the spatial domain was also possible. First results show correlation between the location and certain contact force features. 


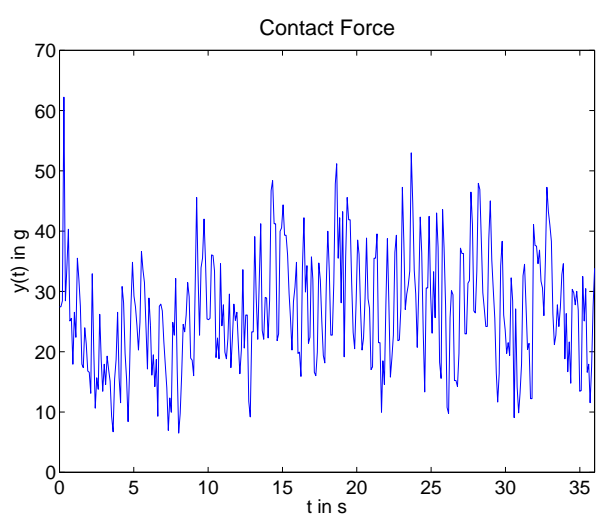

(a)

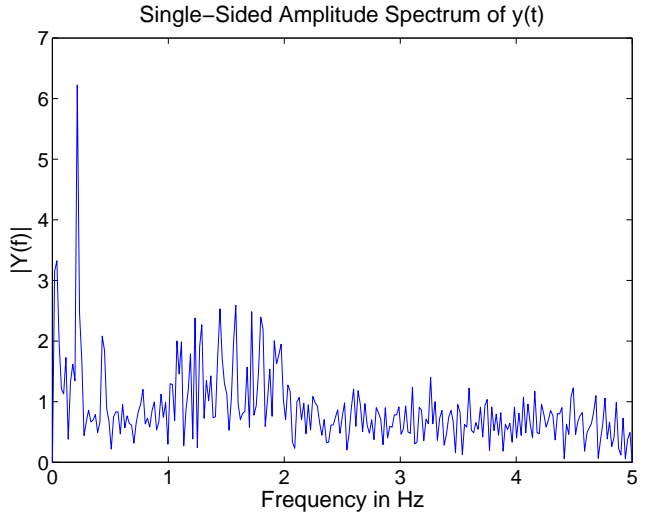

(b)

Figure 3. Contact force signal for a single ablation point. The time domain representation is shown in (a). Figure (b) represents the magnitude of the corresponding signal in frequency domain, with a peak at $0.25 \mathrm{~Hz}$.

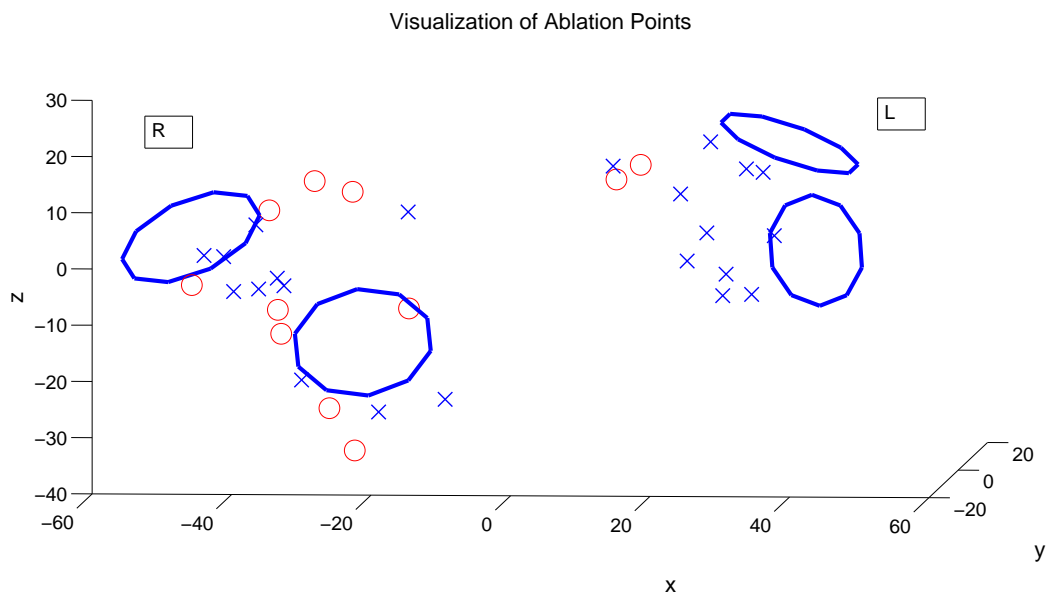

Figure 4. 3-D plot of tracked ablation points in anatomical context. Blue ellipses represent the pulmonary vein ostia of the left atrium. Ablation points tracked during PVI are labeled as follows: Points with high breathing motion influence are represented as a red circle, all others as a blue cross. The axis $\mathrm{x}, \mathrm{y}$ and $\mathrm{z}$ correspond to the sagittal, coronal and axial direction in the patient coordinate system respectively.

In Figure 4, the ablation points for a single ablation procedure are shown. Points with high filter response are highlighted with red circles. Table 1 shows the average values and standard deviation for $p^{m}$, FTI, average contact force and ablation time for each group separately. The case labels 'c01', 'c02' and 'c03' refer to data from three different pulmonary vein isolation (PVI) procedures. The ablation duration is roughly the same in both populations, whereas the force related parameters, FTI and average force, differ considerably.

\section{CONCLUSION}

We investigated the influence of respiratory motion on the catheter tip-to-tissue contact during ablation procedures. Initial findings show that motion patterns can be estimated from the force information. We also found that motion due to respiration is more pronounced on the roof and around the right pulmonary veins, as can be seen in Figure 4. These ablation points also show 


\begin{tabular}{|c|c|c|c|c|c|}
\hline Case & Group & $p^{m}$ & FTI & Avg. force & Time \\
\hline $\mathrm{c} 01$ & (I) & $8.9 \pm 3.8$ & $543.2 \pm 237.4 \mathrm{gs}$ & $16.9 \pm 8.6 \mathrm{~g}$ & $32.5 \pm 7.2 \mathrm{~s}$ \\
\hline $\mathrm{c} 01$ & (II) & $2.8 \pm 1.1$ & $314.8 \pm 215.9 \mathrm{gs}$ & $10.4 \pm 6.6 \mathrm{~g}$ & $28.9 \pm 11.4 \mathrm{~s}$ \\
\hline $\mathrm{c} 02$ & (I) & $6.8 \pm 1.7$ & $759.7 \pm 200.6 \mathrm{gs}$ & $20.3 \pm 6.5 \mathrm{~g}$ & $38.5 \pm 6.5 \mathrm{~s}$ \\
\hline $\mathrm{c} 02$ & (II) & $3.2 \pm 1.1$ & $413.7 \pm 184.6 \mathrm{gs}$ & $12.8 \pm 5.2 \mathrm{~g}$ & $33.6 \pm 10.9 \mathrm{~s}$ \\
\hline $\mathrm{c} 03$ & (I) & $7.8 \pm 1.3$ & $883.8 \pm 197.8 \mathrm{gs}$ & $24.8 \pm 5.9 \mathrm{~g}$ & $35.8 \pm 4.9 \mathrm{~s}$ \\
\hline $\mathrm{c} 03$ & (II) & $2.3 \pm 1.1$ & $459.7 \pm 280.8 \mathrm{gs}$ & $13.4 \pm 5.4 \mathrm{~g}$ & $32.6 \pm 13.1 \mathrm{~s}$ \\
\hline total & (I) & $7.8 \pm 2.8$ & $694.5 \pm 248.1 \mathrm{gs}$ & $19.7 \pm 7.6 \mathrm{~g}$ & $35.6 \pm 6.9 \mathrm{~s}$ \\
\hline total & (II) & $2.6 \pm 1.1$ & $415.9 \pm 246.8 \mathrm{gs}$ & $12.6 \pm 5.7 \mathrm{~g}$ & $32.1 \pm 12.2 \mathrm{~s}$ \\
\hline
\end{tabular}

Table 1. Evaluation of contact force characteristics. The ablation points are grouped based on their respiratory signal response. Points with high respiratory motion influence were added to Group (I), the remaining points were assigned to Group (II). The mean values \pm std. are shown for $p^{m}$, FTI, average contact force and ablation time, respectively. Evaluation has been performed for each procedure data separately (case labels 'c01', 'c02' and 'c03'), as well as over all ablation points combined ('total').

higher average contact force, which directly relates to higher FTI given similar ablation times. Our work showed that contact force increases at regions affected by higher motion. However, further research is needed to verify this hypothesis.

\section{ACKNOWLEDGMENTS}

This work was supported by the German Federal Ministry of Education and Research (BMBF) in the context of the initiative Spitzencluster Medical Valley - Europäische Metropolregion Nürnberg, project grant Nos. 01EX1012A and 01EX1012E, respectively. Additional funding was provided by Siemens AG, Healthcare Sector.

Disclaimer: The concepts and information presented in this paper are based on research and are not commercially available.

\section{References}

[1] R. Weerasooriya, P. Khairy, J. Litalien, L. Macle, M. Hocini, F. Sacher, N. Lellouche, S. Knecht, M. Wright, I. Nault, S. Miyazaki, C. Scavee, J. Clementy, M. Haissaguerre, and P. Jais, "Catheter ablation for atrial fibrillation: Are results maintained at 5 years of follow-up?," Journal of the American College of Cardiology 57(2), 160 - 166 (2011).

[2] F. Ouyang, R. Tilz, J. Chun, B. Schmidt, E. Wissner, T. Zerm, K. Neven, B. Köktürk, M. Konstantinidou, A. Metzner, A. Fuernkranz, and K.-H. Kuck, "Long-term results of catheter ablation in paroxysmal atrial fibrillation / clinical perspective," Circulation 122(23), 2368-2377 (2010).

[3] P. Neuzil, D. Wichterle, R. Cihak, J. Skoda, E. Wissner, O. Fremont, A. Yulzari, V. Y. Reddy, J. Kautzner, and K.-H. Kuck, "EFFICAS I Results - Are Low Contact Force Parameters Predictors for Gap Formation after Pulmonary Vein Isolation by Radiofrequency Catheter Ablation?," in AHA Scientific Sessions 2011 - New Techniques and Technologies for AF Ablation, (2011).

[4] K. McLeish, D. Hill, D. Atkinson, J. Blackall, and R. Razavi, "A study of the motion and deformation of the heart due to respiration," IEEE Transactions on Medical Imaging 21, 1142 -1150 (2002).

[5] J. Ector, S. De Buck, D. Loeckx, W. Coudyzer, F. Maes, S. Dymarkowski, J. Bogaert, and H. Heidbüchel, "Changes in left atrial anatomy due to respiration: Impact on three-dimensional image integration during atrial fibrillation ablation," Journal of Cardiovascular Electrophysiology 19(8), 828-834 (2008).

[6] H. U. Klemm, D. Steven, C. Johnsen, R. Ventura, T. Rostock, B. Lutomsky, T. Risius, T. Meinertz, and S. Willems, "Catheter motion during atrial ablation due to the beating heart and respiration: Impact on accuracy and spatial referencing in three-dimensional mapping," Heart Rhythm 4(5), 587592 (2007). 
[7] S. De Buck, F. Maes, J. Ector, J. Bogaert, S. Dymarkowski, H. Heidbuchel, and P. Suetens, "An augmented reality system for patient-specific guidance of cardiac catheter ablation procedures," IEEE Transactions on Medical Imaging 24(11), 1512-1524 (2005).

[8] F. Bourier, D. Vukajlovic, A. Brost, J. Hornegger, N. Strobel, and K. Kurzidim, "Pulmonary vein isolation supported by MRI-derived 3D-augmented biplane fluoroscopy: A feasibility study and a quantitative analysis of the accuracy of the technique," Journal of Cardiovascular Electrophysiology (2012). [epub ahead of print].

[9] A. Brost, R. Liao, J. Hornegger, and N. Strobel, "3-D Respiratory Motion Compensation during EP Procedures by Image-Based 3-D Lasso Catheter Model Generation and Tracking," in Lecture Notes in Computer Science, G.-Z. Yang, D. J. Hawkes, D. Rueckert, J. A. Noble, and C. J. Taylor, Eds., 5761, 394-401 (2009).

[10] A. Brost, A. Wimmer, R. Liao, F. Bourier, M. Koch, N. Strobel, K. Kurzidim, and J. Hornegger, "Constrained Registration for Motion Compensation in Atrial Fibrillation Ablation Procedures," IEEE Transactions on Medical Imaging 31(4), 870-881 (2012).

[11] S. Kaeppler, A. Brost, M. Koch, W. Wu, F. Bourier, T. Chen, K. Kurzidim, J. Hornegger, and N. Strobel, "Motion Estimation Model for Cardiac and Respiratory Motion Compensation," in Lecture Notes in Computer Science, Purang and J. Abolmaesumi, Eds., 7330, 94-103, (Berlin Heidelberg) (2012).

[12] K. Yokoyama, H. Nakagawa, D. Shah, H. Lambert, G. Leo, N. Aeby, A. Ikeda, J. Pitha, T. Sharma, R. Lazzara, et al., "Novel Contact Force Sensor Incorporated in Irrigated Radiofrequency Ablation Catheter Predicts Lesion Size and Incidence of Steam Pop and Thrombus," Circulation: Arrhythmia and Electrophysiology 1(5), 354 (2008).

[13] E. Wissner, J. Petru, A. Metzner, P. Peichel, M. Soranno, H. Lambert, J. Kautzner, P. Neuzil, and K.-H. Kuck, "The EFFICAS studies: Reducing low force-time integral (FTI) radiofrequency applications improves procedural efficacy during pulmonary vein isolation," in AHA Scientific Sessions 2011 - New Techniques and Technologies for AF Ablation, (2011).

[14] M. Koch, A. Langenkamp, A. Kiraly, A. Brost, N. Strobel, and J. Hornegger, "Navigation system with contact force assessment to guide pulmonary vein isolation procedures," in 23rd Conference of the Society for Medical Innovation and Technology (SMIT), (Tel Aviv, Israel) (2011).

[15] M. Koch, A. Brost, A. Kiraly, N. Strobel, and J. Hornegger, "Post-procedural evaluation of catheter contact force characteristics," in SPIE Medical Imaging, SPIE, Ed., 8315, 83152J (2012).

[16] R. Bailon, L. Sornmo, and P. Laguna, "A robust method for ecg-based estimation of the respiratory frequency during stress testing," IEEE Transactions on Biomedical Engineering 53(7), 1273 -1285 (2006).

[17] P. Novak and V. Novak, "Time/frequency mapping of the heart rate, blood pressure and respiratory signals," Medical and Biological Engineering and Computing 31, 103-110 (1993).

[18] D. Shah, H. Lambert, H. Nakagawa, A. Langenkamp, N. Aeby, and G. Leo, "Area under the real-time contact force curve (force-time integral) predicts radiofrequency lesion size in an in vitro contractile model," Journal of Cardiovascular Electrophysiology 21(9), 1038-1043 (2010). 